\title{
Análise Comparativa da Geração de Fumos entre Arames Maciços (GMAW) e Tubulares (FCAW)
}

\author{
(Comparative Analysis of Fume Generation between solid (GMAW) and flux cored wires (FCAW))
}

\author{
Regina Paula Garcia ${ }^{1}$, Américo Scotti ${ }^{1}$ \\ ${ }^{1}$ Universidade Federal de Uberlândia, Centro para Pesquisa e Desenvolvimento de Processos de Soldagem - Laprosolda - da \\ Faculdade de Engenharia Mecânica, Uberlândia, Minas Gerais, Brasil, regisrpg@yahoo.com.br; ascotti@ufu.br
}

\begin{abstract}
Resumo
O processo GMAW (MIG/MAG) é muito utilizado no meio industrial. Entretanto, este processo apresenta limitações quanto a ações metalúrgicas sobre a zona fundida e moldabilidde do cordão. O processo FCAW, por outro lado, por apresentar elementos fluxantes, consegue melhores resultados nestes aspectos, mas o fluxo pode ser um agente responsável por elevada emissão de fumos. Este trabalho teve por objetivo apresentar um estudo comparativo da geração de fumos entre o processo GMAW com transferência metálica por curto-circuito e o processo FCAW sob proteção gasosa, ambos com eletrodos de mesma classe de resistência mecânica (483 MPa de limite de ruptura) e aplicação. Os experimentos foram realizados utilizando-se um coletor de fumos calibrado de acordo com norma. Os dois consumíveis foram avaliados sob uma proteção de $100 \% \mathrm{CO}_{2}$ em dois níveis de corrente. O efeito do comprimento do arco e do uso de outra proteção $\left(\right.$ Ar $\left.+25 \% \mathrm{CO}_{2}\right)$ também foi avaliado. Para se possibilitar uma comparação próxima da realidade prática de cada consumível, procurou-se trabalhar com cada combinação aramelgás de proteção em condições apropriadas de distância bico de contato-peça e de regulagem de tensão. Fixou-se ainda o mesmo volume do cordão de solda por metro para cada nível de corrente, mantendo-se constante a relação entre a taxa de deposição e a velocidade de soldagem. Os resultados confirmam uma maior taxa de geração de fumos pelo processo FCAW (já esperado) e que a taxa absoluta de fumos cresce com o aumento da corrente de soldagem, porém para processo GMAW analisado de forma relativa (em função da massa fundida) isso não ocorre. Verificou-se que o efeito da corrente é mais significativo para o processo FCAW. O aumento do teor de $\mathrm{CO}_{2}$ elevou a taxa de geração de fumos apenas no processo FCAW, enquanto que a variação da tensão de arco não demonstrou qualquer efeito sobre esta.
\end{abstract}

Palavras-chave: fumos; GMAW; FCAW; arame tubular; proteção gasosa.

Abstract: The GMAW (MIG /MAG) process is widespread in industry. However, it presents limitations regarding metallurgical aspects and geometrical characteristics of the weld bead. The FCAW process, on the other hand, due to presence of fluxes, achieves improvements on these aspects, but the flux might be responsible for larger fume emission. This paper aimed to present a comparative study of fume generation between the GMAW process with short-circuit metal transfer and the gas shielded FCAW process, using wires of same class of resistance (483 MPa of ultimate tension) and application. The experiments were performed in a fume chamber calibrated according to a standard. Both wires were evaluated using $100 \% \mathrm{CO}_{2}$ as the shielding gas and at two current levels. The arc length effect and the use of another shielding gas (25\% $\mathrm{CO}_{2}$ in argon) were also evaluated. Appropriate conditions of contact-tip-to-work-piece distance and arc voltage were selected for each wire-shielding gas combination as a means of making the comparison closer to the reality. The volume of the weld beads per meter was made the same for each level of current, by keeping the same relationship between wire deposition and welding travel speed. The results confirm a higher fume generation rate of the FCAW process (already expected) and that absolute fume generation rate grows as the welding current increases. However, for the GMAW process, analyzed from a relative point of view, it doesn't happen. It was verified that the current effect is more significant on the FCAW process. The increase in the CO ${ }_{2}$ content raised the fume generation rate only for the FCAW process, whilst the arc voltage variation did not demonstrate any effect on it.

Key-words: Fumes, GMAW; FCAW; tubular wire; gas shielding.

\section{Introdução}

O estudo e a análise da geração de fumos possuem um papel importante no que se refere à soldagem, uma vez que normas

(Recebido em 20/11/2009; Texto final em 28/05/2010). Artigo originalmente publicado no CONSOLDA 2009, Piracicaba, SP, Outubro de 2009. rígidas de Segurança do Trabalho determinam o tempo e o limite de exposição do soldador aos fumos. Desse modo, minimizar a taxa de geração de fumos e dos riscos à saúde do trabalhador é sempre uma preocupação, uma vez que este é o responsável pelo alto índice de doenças respiratórias e doenças que afetam o pulmão entre os soldadores [1]. É possível minimizar a quantidade de fumos gerados, aos quais os soldadores estão expostos, avaliando-se uma série de fatores operacionais, tais como seleção adequada de parâmetros de soldagem, eficiência do processo de soldagem de acordo com sua aplicação, utilização da ventilação mais adequada para o ambiente de 
soldagem, o material de adição e os gases de proteção [2]. Entretanto, a procura por otimização dos processos de soldagem no ambiente industrial tem tido como objetivo principal elevar os índices de produtividade, aliados à redução de custos. Nesta perspectiva, encontram-se processos de alta produtividade (alta produção a baixo custo), tais como o GMAW, que tem dominado soberanamente o mundo da união dos metais por soldagem. Porém, o processo FCAW tem se despontado como uma alternativa para suprir as deficiências do GMAW, garantindo a mesma eficiência no que tange a produtividade. Mas pouco se tem demonstrado da relação entre esta produtividade obtida com a geração de fumos destes processos/técnicas.

De acordo com Quimby e Ulrich [3], a realização de estudos sobre a taxa de geração de fumos (TGF, referenciada em inglês como FGR - Fume Generation Rate) no processo GMAW é complexa e influenciada por uma série de variáveis, dificultando a realização de uma análise comparativa entre os resultados disponíveis, inclusive de propor modelos teóricos que expliquem o aumento da TGF. Estudos revelam que o aumento da corrente aumenta a taxa de geração de fumos [2,4], isso, conforme os autores, como consequiência de um aumento da temperatura do arco, uma vez que proporciona uma maior vaporização de metais presentes no arco (outro fator seria apontado pelos autores deste trabalho, porque há um aumento da taxa de fusão do eletrodo por unidade de tempo). É importante frisar que para outros pesquisadores, como, por exemplo, Lancaster [5], o aumento da corrente não aumenta a temperatura do arco, ficando a segunda explicação mais plausível. Segundo Jenkins[6], para o processo GMAW o fumo é predominantemente oriundo da evaporação da ponta do eletrodo, isso ocorrendo porque a temperatura nessa região é maior do que na poça de fusão, devido a alta densidade de calor. Para Castner[2], o modo de transferência metálica influencia na taxa de geração de fumos. Para Jenkins[6], as transferências por curto circuito e spray produzem menores níveis de fumos do que a transferência globular, em função das gotas menores (curto-circuito e spray) possuírem uma menor área superficial e, portanto, absorverem uma menor quantidade de calor do que gotas grandes (globular). A tensão é outro parâmetro capaz de alterar a taxa de fumos, segundo Castner [2] e Yamame [4]. Segundo estes autores, o aumento da tensão aumenta a taxa de fumos, pois ocorre uma maior liberação de vapores a altas temperaturas do arco para atmosfera, pois com o aumento da tensão há um aumento da velocidade do fluxo de plasma. De acordo com Gray et al [7] apud Quimby e Ulrich [3] estudos realizados com o processo GMAW com aços inoxidáveis, revelam que o aumento da TGF em função do aumento da tensão apresenta uma descontinuidade, conforme Figura 1, mas em razão da variação do modo de transferência e não da tensão em si.

A composição do gás de proteção também afeta a geração de fumos para o processo GMAW, como ilustra a Figura 2. Procedimentos de soldagem que utilizem como gás de proteção $100 \%$ de $\mathrm{CO}_{2}$ produz mais fumos e respingos do que quando se utilizam misturas de argônio [2,3], em função da diferença do potencial de oxidação dos gases. Também verifica-se que o aumento do teor de $\mathrm{CO}_{2}$ no gás de proteção resulta num aumento da condutividade térmica do gás [8] e então para manter o mesmo nível de corrente requer uma faixa de tensão de trabalho maior do que quando se utiliza misturas de argônio. Pode-se supor que, de certa forma, todos os parâmetros que são capazes de alterar a taxa de fumos influenciam diretamente na fusão do eletrodo, ou no modo de transferência metálica.

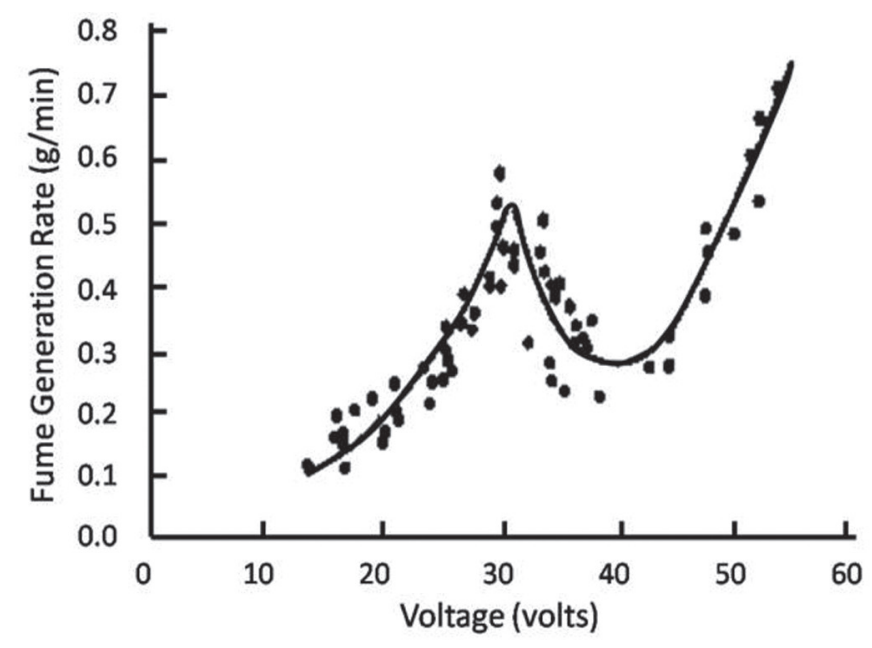

Figura 1. TGF (Fume generation rate) para diferentes modos de transferência metálica para um aço inoxidável, destacando-se o aumento da tensão no processo GMAW na mudança do modo de transferência (Gray et al [7] apud

Quimby e Ulrich [3])

Todas estas análises de parâmetros que influenciam a geração de fumos referem se ao processo GMAW, sobre o qual se possui uma vasta literatura. Já para o processo FCAW, as fontes de referência são mais restritas, mas é um fato que este processo apresenta uma taxa de geração de fumos maior que no primeiro processo citado [3,4]. Segundo Rosado et al [9], arames tubulares produzem uma taxa maior de fumos para uma mesma intensidade de corrente do que arames do tipo "metalcored", uma vez que o efeito resistivo no arame tubular é maior do que no "metal-cored", resultando num maior aquecimento e evaporação do fluxo. Por outro lado, o processo FCAW apresenta ótimas características operacionais e uma maior versatilidade para utilização em campo. A composição química do fluxo permite alterações que influencia diretamente nas características geométricas do cordão e nas propriedades mecânicas do mesmo.

Com o crescimento da utilização do processo FCAW, torna-se necessário compreender as nuances da geração de fumos do processo para atender os limites especificados por normas de segurança e saúde ocupacional, que visam fornecer aos trabalhadores condições adequadas de trabalho. Bem como avaliar todos os parâmetros que são capazes de alterar o desempenho operacional do processo. Mas considerando as dificuldades de se determinar com precisão a taxa de geração de fumos, torna-se importante o estabelecimento de uma metodologia para avaliar comparativamente o processo GMAW e FCAW, no que tange a geração de fumos, incluindo o efeito dos parâmetros de cada processo que são capazes de influenciar na geração da taxa de fumos. Poder-se-ia, assim, consolidar um roteiro prático que orientasse os usuários dos dois processos. Desta forma, o objetivo deste trabalho é uma comparação entre os 
dois processos, sob diferentes correntes, tipos de gás de proteção e tensões de soldagem, usando-se uma metodologia que procure atingir valores comparáveis e que seus resultados, notadamente os efeitos de cada variável estudada, sejam passíveis de uso pelos usuários de soldagem.

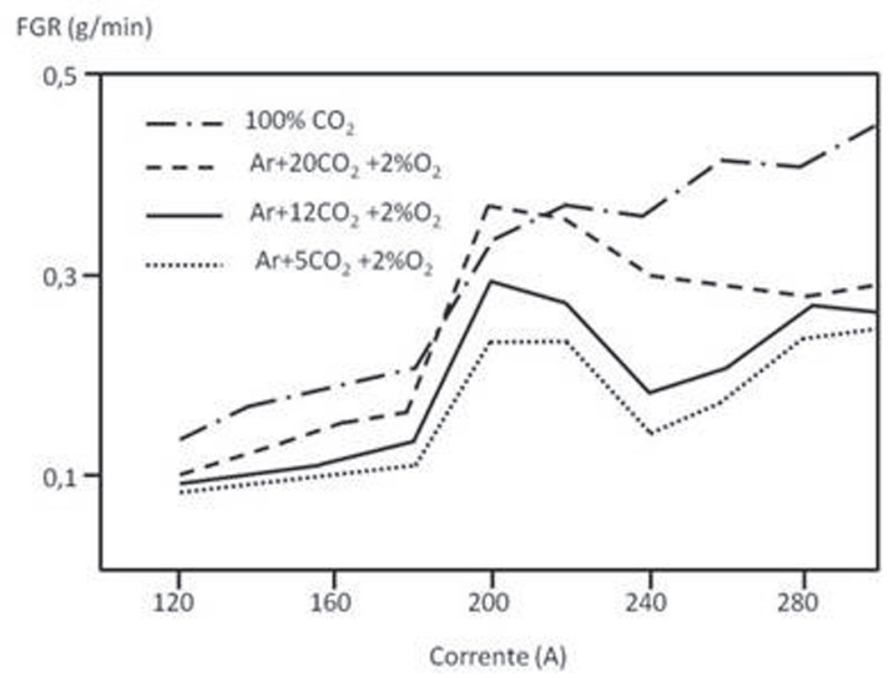

Figura 2. Taxa de geração de fumo em função do gás de proteção para GMAW (adaptado Castner [2])

\section{Matérias e Métodos}

Realizou uma série de testes, tomando como base o dispositivo e metodologia para medição de fumos estabelecida pela norma AWS F1.2:2006 [10]. Para a coleta do fumo na câmara coletora, foi utilizado um filtro de fibra de vidro especificado conforme ASTM C800[11], a qual determina a temperatura e espessura adequadas para o manuseio do filtro. A citada câmara coletora de fumos, ilustrada pela Figura 3, foi devidamente calibrada conforme descrito na referida norma. O padrão de calibração da norma prevê a utilização do eletrodo ER70S-3, com diâmetro de $1,2 \mathrm{~mm}$, tensão de $24 \mathrm{~V}$, corrente de aproximadamente 225 A, uma distância bico contato-peça (DBCP) de 19 mm, gás de proteção $100 \% \mathrm{CO}_{2}$, com uma vazão de 18 1/min e velocidade de alimentação do arame de 7,6 m/min. A execução da calibração do equipamento com estes parâmetros levou a uma taxa de fumos $0,35 \mathrm{~g} / \mathrm{min}$, um valor aceitável pelo estabelecido pela norma que prevê uma margem de erro de $10 \%$ para reprodutibilidade do ensaio. Todo o procedimento para a execução do ensaio de coleta de fumos está descrito no procedimento interno do Laprosolda [12], que visa padronizar internamente a realização dos ensaios de coleta de fumos, independente da finalidade de tais testes.

Os equipamentos necessários para a realização do ensaio são:

a) Fonte de soldagem inversora, regulada no modo de trabalho tensão constante;

b) Cilindro de gás com regulador de pressão, para controle da vazão de gás;

c) Balança de precisão, calibrada, com resolução de 0,01 g, para quantificar a coleta dos fumos;

d) Estufa para ressecamento dos filtros, com faixa de trabalho entre $0^{\circ} \mathrm{C}$ e $150{ }^{\circ} \mathrm{C}$; e) Termômetro (analógico ou digital), calibrado, para controle da temperatura da estufa;

f) Cronômetro digital, para controle do tempo de execução do teste;

g) Sistema de aquisição e tratamento de dados, para monitorar os valores de corrente, tensão e velocidade de alimentação de arame, composto por um microcomputador com software de aquisição de sinais, um conversor A/D com comunicação USB para a aquisição, uma interface de proteção e tratamento de sinais elétricos e um tacômetro com saída analógica.

h) Unidade robótica, para garantir a reprodutibilidade da velocidade de soldagem.

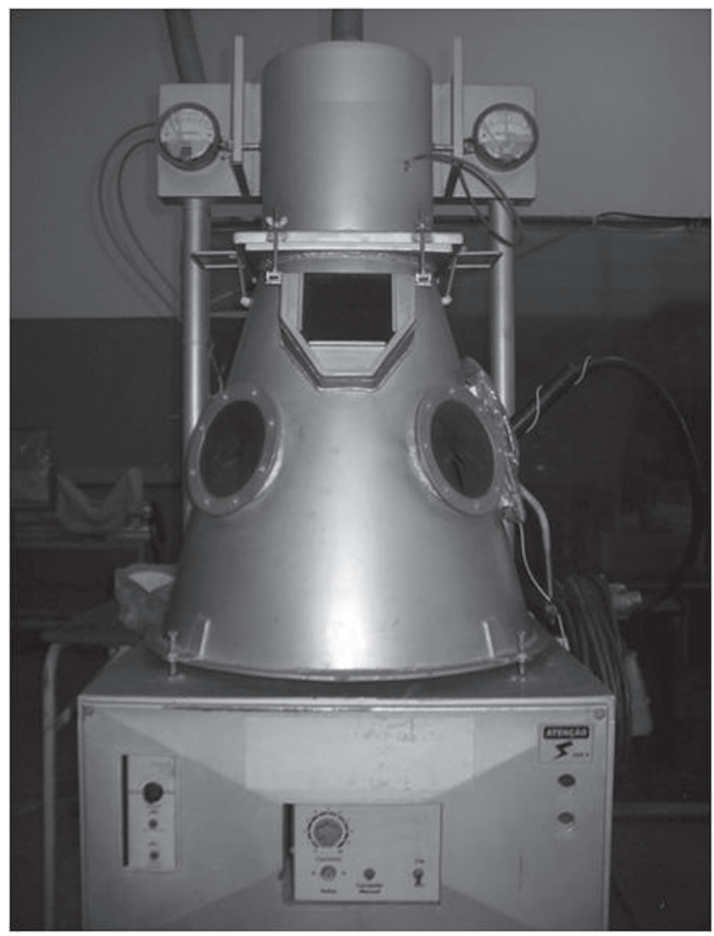

Figura 3- Equipamento para a coleta de fumos e medição da taxa de geração de fumos (TGF)

Do ponto de vista metodológico, os resultados de emissão de fumos podem ser avaliados sob dois ângulos. O primeiro, referente ao aspecto da capacidade maior ou menor de um consumível em gerar fumos. Desta forma, torna-se importante expressar os resultados como massa de fumos coletadas por unidade de massa de arame consumido, como recomendado por Rosado et al. [9]. O segundo ângulo se refere à exposição do soldador aos fumos, o que, a princípio, requereria os resultados expressos como massa de fumo coletado por unidade de tempo. Seja por um ângulo ou por outro, acredita-se que uma comparação entre os consumíveis só seria correta se realizada para um mesmo volume de cordão solda, pois, afinal, uma comparação teria de ter como base a realização de um mesmo trabalho (um mesmo volume de cordão representaria preencher um mesmo chanfro, qualquer que seja ele). Entretanto, no caso da exposição ao fumo, aplicar-se-ia uma nova correção, pois um material que produza uma grande quantidade de fumos por unidade de tempo, mas gaste menos tempo para ser fazer uma soldagem, pode ser menos problemático. Assim, entendeu-se neste trabalho que o 
resultado correto do ponto de vista de exposição seria massa de fumos coletado por unidade de comprimento de solda (já que os volumes são iguais).

Para a realização do estudo comparativo entre os processos, utilizou se os consumíveis arame maciço ER70S-6 e arame tubular E71T-1, ambos com gases de proteção de $100 \% \mathrm{CO}_{2}$ e também de uma mistura $75 \% \mathrm{Ar}+25 \% \mathrm{CO}_{2}$. Como placa de teste, foi utilizado um disco de aço ABNT 1020, com 12,7 mm de espessura e com $400 \mathrm{~mm}$ de diâmetro. Cada condição de teste foi realizada no mínimo três vezes, para garantir uma maior significância estatística dos resultados de taxa de fumos gerados. Quando havia dispersão de um dos 3 resultados, era repetido o teste até que se alcançassem três resultados com pequena variância, rejeitando-se os valores díspares (em alguns casos foi necessário medir 5 vezes a mesma condição). Para o arame maciço foi utilizada uma distância bico de contato-peça (DBCP) de $12 \mathrm{~mm}$ e uma vazão de gás de 15 litros por minuto. Para o tubular, uma DBCP de $20 \mathrm{~mm}$ com uma vazão de 20 litros por minuto foi aplicada. Estas condições são típicas para estes consumíveis.

Como os dois processos apresentam diferenças significativas no desempenho operacional, principalmente no que tange a taxa de fusão e modo de transferência, procurou-se encontrar uma forma de se efetuar uma comparação baseada nas seguintes premissas:

1) Fixaram-se dois níveis de corrente para os testes $(150 \pm 5$ A, considerado um valor médio para uma faixa de baixa corrente, e $250 \pm 5 \mathrm{~A}$, considerado um valor médio para uma faixa de alta corrente para ambos os processos), tomando com base a importância deste parâmetro na geração de fumos e o efeito da corrente sobre o modo de transferência, que também é um parâmetro de significância;

2) Fixou-se a mesma taxa de deposição por unidade de comprimento, uma para cada valor de corrente (já que não foi possível, para os valores iniciais já obtidos em $150 \mathrm{~A}$, manter um cordão adequado com uma mesma taxa usando $250 \mathrm{~A}$ ), evitando o efeito de volumes diferentes de poça, que poderia também afetar a taxa de geração de fumos.

Para determinar os parâmetros de soldagem (velocidade de alimentação, tensão de regulagem e velocidade de soldagem) para ambos os processos que fornecessem a mesma taxa de deposição por unidade de comprimento e resultasse num cordão visualmente aceitável para os processos, foi realizado o seguinte procedimento:

a) Foram realizados testes preliminares com o arame tubular, adotando: 1) os parâmetros recomendados pelo fabricante (valor de distância bico de contato-peça e a faixa de tensão de trabalho); 2) uma velocidade de alimentação $\left(\mathrm{V}_{\mathrm{al}}\right)$ que levasse à corrente desejada; 3) uma faixa e tensão de regulagem para a qual esperava se soldagens estáveis para cada corrente; e 4) uma velocidade de soldagem $\left(\mathrm{V}_{\text {sol }}\right)$ que fornecesse um cordão de solda com volume condizente e bom aspecto visual. Nestes testes, procurou-se também, por varredura, uma faixa de tensão de regulagem que resultasse em cordões de bom aspecto e definiu-se o valor central da faixa como a regulagem para soldagem $\left(\mathrm{U}_{\text {reg }}\right)$. Estes testes foram, assim, realizados a fim de se obter os parâmetros de regulagem (tensão, velocidade de alimentação e velocidade de soldagem) que resultassem num cordão adequado com o FCAW;

b) Determinou-se a taxa de deposição por unidade de tempo $\left(T_{d}\right)$ do eletrodo tubular, para cada corrente, a partir da diferença de massa da chapa antes e após soldada, Equação 1 , e, em seguida, a taxa de deposição por unidade de comprimento $\left(T_{D}\right)$, expressa pela Equação 2, obtendo os valores $T_{D}$ de $1,32 \mathrm{~g} / \mathrm{cm}$ para $150 \mathrm{~A} \mathrm{e}_{\mathrm{D}}$ de $1,77 \mathrm{~g} / \mathrm{cm}$ para $250 \mathrm{~A}$ (com a Equação 3, é possível também determinar o rendimento de deposição, $\mathrm{h}_{\mathrm{D}}$ );

c) Fixou-se a $T_{D}$ encontrada para o arame tubular como referência a ser atingida com o arame maciço nos testes práticos e, assim, atingir o mesmo volume de solda;

d) Calculou-se a taxa de fusão $\left(T_{F}\right)$ de arame maciço, para cada nível de corrente, a partir da Eq. 4 (para se determinar a densidade do arame, efetuaram-se medições de diâmetro do arame ao longo de dois metros, utilizando-se um micrômetro, para calcular o volume e, através de pesagem, obtevese a massa para esta quantidade de arame, o que resultou numa densidade de $7,85 \mathrm{~g} / \mathrm{cm}^{3}$ ). $\mathrm{O}$ mesmo procedimento foi realizado para o arame tubular, o que resultou numa densidade de $6,40 \mathrm{~g} / \mathrm{cm}^{3}$;

e) Assumindo-se hipoteticamente $u m h_{D}$ de $95 \%$ para o arame maciço, obteve-se a primeira aproximação da velocidade de soldagem $\left(\mathrm{V}_{\text {sol }}\right)$ e, a partir da velocidade alimentação para a dada corrente já levantada previamente e do rearranjo da Equação 5, e fez-se uma soldagem com o GMAW para se obter o rendimento real (no caso, $\mathrm{h}_{\mathrm{D}}$ de $98 \%$ ) para essas condições;

f) Realizaram-se soldagens exploratórias com o arame maciço para se determinar a tensão de regulagem $\left(\mathrm{U}_{\text {reg }}\right)$, também por varredura, dentro de uma faixa em que se era esperada bons resultados. Trabalhando com o valor médio da faixa de tensão de regulagem, recursivamente, encontrou-se a $\left(\mathrm{V}_{\text {sol }}\right)$ compatível com a $\left(\mathrm{V}_{\mathrm{al}}\right)$ do arame maciço para cada corrente, de forma a reproduzir o volume depositado pelo arame tubular nas suas regulagens de $\mathrm{V}_{\mathrm{sol}}$ e $\mathrm{V}_{\mathrm{al}}$;

$$
\begin{array}{ll}
T_{d}=\frac{\left(M_{f}-M_{i}\right)}{T_{a b}} & {[\mathrm{~g} / \mathrm{min}]} \\
T_{D}=\frac{T_{d}}{V_{\text {sol }}} & {[\mathrm{g} / \mathrm{cm}]} \\
N_{D}=\frac{T_{d}}{T_{F}} & (\%) \\
T_{F}=\left(\frac{\pi d^{2}}{4}\right) * V_{a l} * \rho & {[\mathrm{g} / \mathrm{min}]} \\
T_{D}=\left(N_{D} * T_{F}\right) / V_{\text {sol }} & {[\mathrm{g} / \mathrm{cm}]}
\end{array}
$$


Tabela 1. Valores de regulagem dos parâmetros e valores médios monitorados

\begin{tabular}{|c|c|c|c|c|c|c|c|c|}
\hline & \multirow[b]{3}{*}{ Teste } & \multirow[b]{3}{*}{ Gás/Corrente } & \multirow{2}{*}{\multicolumn{3}{|c|}{ Regulados }} & \multirow{2}{*}{\multicolumn{3}{|c|}{ Monitorados }} \\
\hline & & & & & & & & \\
\hline & & & $\mathrm{U}_{\mathrm{reg}}(\mathrm{V})$ & $\mathrm{V}_{\mathrm{al}}(\mathrm{m} / \mathrm{min})$ & $\mathrm{V}_{\mathrm{sol}}(\mathrm{cm} / \mathrm{min})$ & $\mathrm{U}(\mathrm{V})$ & $\mathrm{I}(\mathrm{A})$ & $\mathrm{V}_{\mathrm{al}}(\mathrm{m} / \mathrm{min})$ \\
\hline \multirow{4}{*}{ 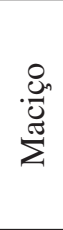 } & 1 & $100 \% \mathrm{CO}_{2} / 150 \mathrm{~A}$ & 21 & 3,20 & 20,14 & 20,8 & 151,5 & 3,2 \\
\hline & 2 & $100 \% \mathrm{CO}_{2} / 250 \mathrm{~A}$ & 24 & 6,80 & 32,23 & 23,8 & 254,8 & 6,8 \\
\hline & 3 & $\mathrm{Ar}+25 \% \mathrm{CO}_{2} / 150 \mathrm{~A}$ & 19 & 2,90 & 19,03 & 19,5 & 149,5 & 3,0 \\
\hline & 4 & $\mathrm{Ar}+25 \% \mathrm{CO}_{2} / 250 \mathrm{~A}$ & 21 & 6,60 & 30,46 & 20,7 & 253,1 & 6,6 \\
\hline \multirow{4}{*}{ 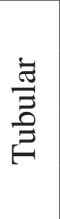 } & 5 & $100 \% \mathrm{CO}_{2} / 150 \mathrm{~A}$ & 26 & 5,00 & 25,00 & 25,7 & 152,7 & 5,0 \\
\hline & 6 & $100 \% \mathrm{CO}_{2} / 250 \mathrm{~A}$ & 30 & 10,70 & 39,00 & 29,5 & 254,8 & 10,6 \\
\hline & 7 & $\mathrm{Ar}+25 \% \mathrm{CO}_{2} / 150 \mathrm{~A}$ & 26 & 4,60 & 23,00 & 25,7 & 154,1 & 4,6 \\
\hline & 8 & $\mathrm{Ar}+25 \% \mathrm{CO}_{2} / 250 \mathrm{~A}$ & 30 & 9,70 & 35,00 & 29,5 & 251,3 & 9,7 \\
\hline
\end{tabular}

Tabela 2. Taxas de geração de fumos resultantes por massa de arame fundido $\left(\mathrm{TGF}_{1}\right)$, por unidade de tempo de arco aberto $\left(\mathrm{TGF}_{2}\right)$ e por unidade de comprimento de solda $\left(\mathrm{TGF}_{3}\right)$

\begin{tabular}{|c|c|c|c|c|c|}
\hline & Teste & Gás/Corrente & $\mathrm{TGF}_{1}(\mathrm{mg} / \mathrm{kg})$ & $\mathrm{TGF}_{2}(\mathrm{~g} / \mathrm{min})$ & $\mathrm{TGF}_{3}(\mathrm{~g} / \mathrm{m})$ \\
\hline \multirow{4}{*}{ 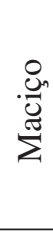 } & 1 & $100 \% \mathrm{CO}_{2} / 150 \mathrm{~A}$ & 6659,00 & 0,18 & 0,89 \\
\hline & 2 & $100 \% \mathrm{CO}_{2} / 250 \mathrm{~A}$ & 4789,00 & 0,27 & 0,85 \\
\hline & 3 & $\mathrm{Ar}+25 \% \mathrm{CO}_{2} / 150 \mathrm{~A}$ & 5555,50 & 0,14 & 0,74 \\
\hline & 4 & $\mathrm{Ar}+25 \% \mathrm{CO}_{2} / 250 \mathrm{~A}$ & 5400,50 & 0,29 & 0,97 \\
\hline \multirow{4}{*}{$\frac{\vec{\Xi}}{\vec{\Xi}}$} & 5 & $100 \% \mathrm{CO}_{2} / 150 \mathrm{~A}$ & 8201,50 & 0,29 & 1,18 \\
\hline & 6 & $100 \% \mathrm{CO}_{2} / 250 \mathrm{~A}$ & 9338,30 & 0,71 & 1,83 \\
\hline & 7 & $\mathrm{Ar}+25 \% \mathrm{CO}_{2} / 150 \mathrm{~A}$ & 6305,40 & 0,21 & 0,91 \\
\hline & 8 & $\mathrm{Ar}+25 \% \mathrm{CO}_{2} / 250 \mathrm{~A}$ & 7296,60 & 0,51 & 1,45 \\
\hline
\end{tabular}

Onde:

$\mathrm{h}_{\mathrm{D}}$ - rendimento de deposição (\%).

${ }^{\mathrm{D}} T_{F}$ - Taxa de fusão do eletrodo, [g/min].

$V_{\text {sol }}$ - velocidade de soldagem, $[\mathrm{cm} / \mathrm{min}]$.

$V_{a l}$ - velocidade de alimentação do arame, [cm/min].

$\rho$ - densidade do arame, $\left[\mathrm{g} / \mathrm{cm}^{3}\right]$.

$M_{f}$ - massa final [g].

$M_{i}$ - massa inicial [g].

$T_{a b}$ - tempo de arco aberto [min].

\section{Resultados e Discussão}

A Tabela 1 apresenta os parâmetros de regulagem e os valores médios monitorados na primeira bateria de testes, permitindo-se verificar que os dois níveis de corrente foram obtidos dentro dos níveis de tolerância estabelecidos e os valores médios monitorados dos parâmetros estão conforme previsto pela metodologia proposta. Já a Tabela 2 apresenta as taxas de geração de fumos do ponto de vista de avaliação da capacidade de geração de um consumível em relação ao outro (ou seja, massa gerada por massa fundida, identificada aqui por $\mathrm{TGF}_{1}$ ) e também sobre a óptica de exposição do soldador (massa gerada por unidade de tempo, $\mathrm{TGF}_{2}$, e por unidade de comprimento de solda, $\mathrm{TGF}_{3}$ ), sendo que estes resultados são representado graficamente nas Figuras 4, 7 e 8.

A Figura 4 permite a análise comparativa para os dois arames, do ponto de vista da capacidade de cada consumível em gerar fumos. É apresentada a TGF em mg/kg, como função da corrente e sob influência dos dois gases de proteção (100\% $\mathrm{CO}_{2}$ e $\mathrm{Ar}+25 \% \mathrm{CO}_{2}$ ). Nesta figura, fica evidente que o eletrodo tubular produz fumos em taxas maiores, o que já era previsto. Entretanto, ao se usar esta figura para se avaliar os efeitos do gás de proteção e da corrente, os comportamentos são diferentes entre o FCAW e o GMAW. Observa-se a ocorrência de um incremento na taxa de geração de fumos com o aumento da corrente para o processo FCAW, fato provavelmente devido a um aumento na taxa de fusão, onde há uma maior evaporação de material fundido oriundo da ponta do eletrodo, conforme mostram dados da literatura $[4,6]$. Porém, para o processo GMAW não ocorreu um aumento da TGF em $\mathrm{mg} / \mathrm{kg}$ com a elevação da corrente.

Antes de se analisar o comportamento apontado no parágrafo acima, procurou-se analisar as diferenças de transferência entre a condição de $150 \mathrm{~A}$ e a de $250 \mathrm{~A}$, tomando-se como 


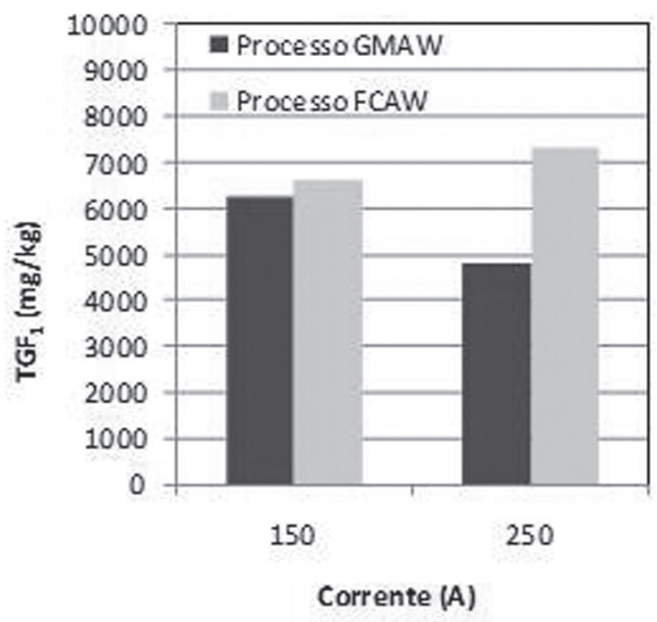

(a)

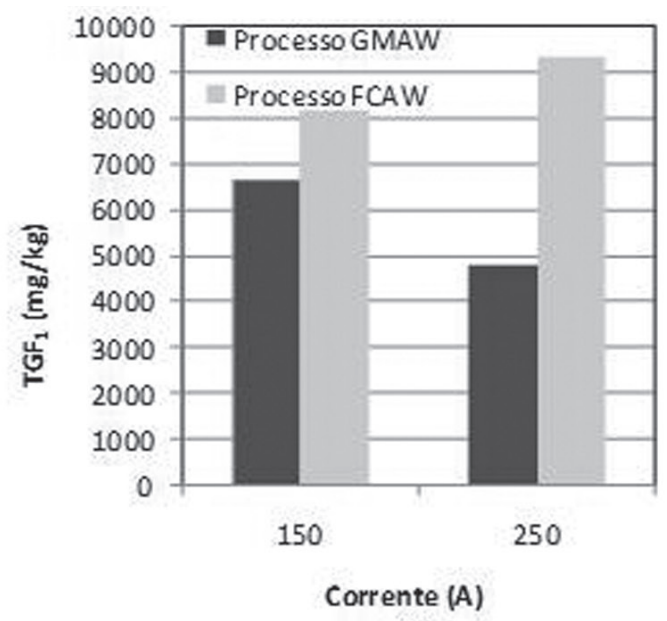

(b)

Figura 4. Comparação da $\mathrm{TGF}_{1}\left(\mathrm{mg} / \mathrm{kg}\right.$ ) para o processo GMAW e FCAW: (a) Para gás de proteção $\mathrm{Ar}+25 \% \mathrm{CO}_{2}$; e (b) para gás de proteção $100 \% \mathrm{CO}_{2}$

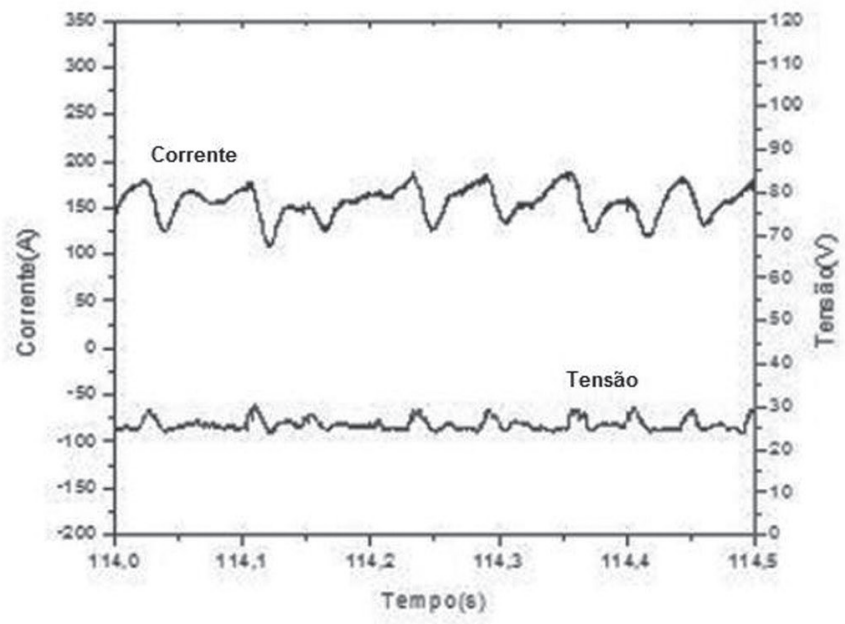

(a)

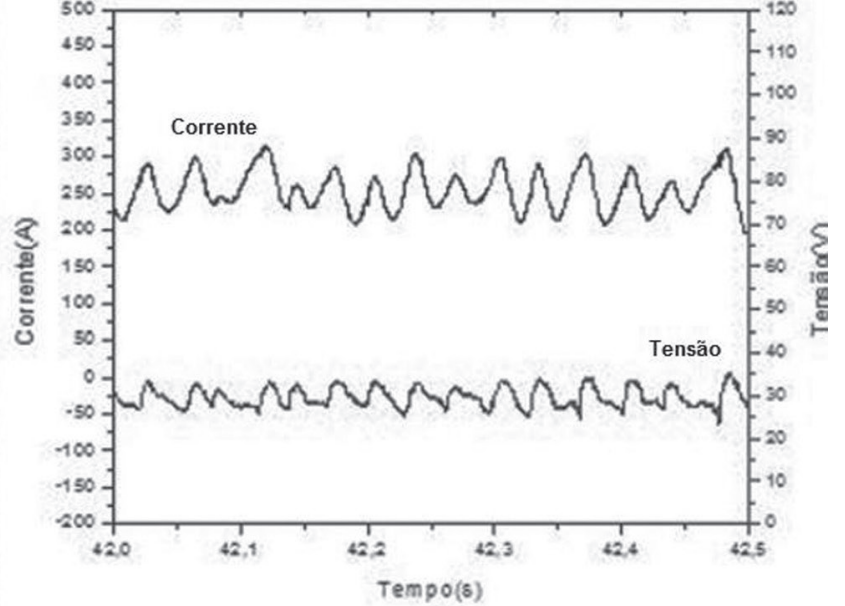

(b)

Figura 5. Oscilogramas de corrente e tensão para o processo FCAW com gás de proteção $100 \% \mathrm{CO}_{2}$ : a) Corrente de $150 \mathrm{~A}$ e tensão regulada $26 \mathrm{~V}$; b) Corrente $250 \mathrm{~A}$ e tensão regulada $30 \mathrm{~V}$

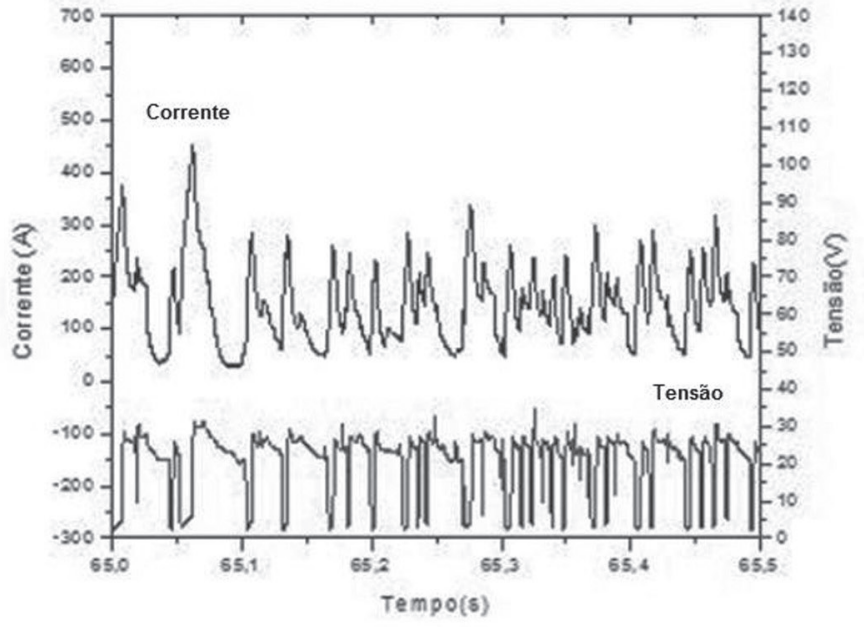

(a)

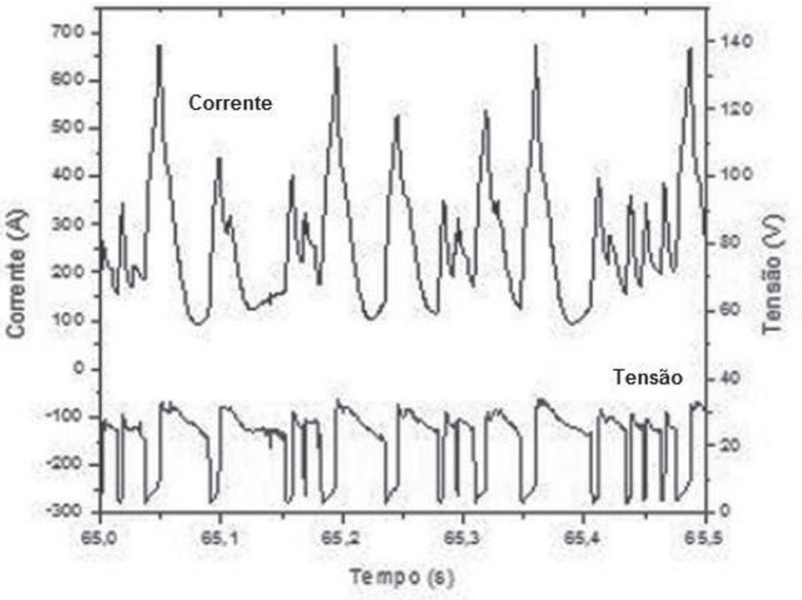

(b)

Figura 6. Oscilogramas de corrente e tensão para o processo GMAW com gás de proteção $100 \% \mathrm{CO}_{2}$ : a) Corrente de $150 \mathrm{~A}$ e tensão regulada $21 \mathrm{~V}$; b) Corrente $250 \mathrm{~A}$ e tensão regulada $24 \mathrm{~V}$ 
base a proteção mais crítica, com $100 \% \mathrm{CO}_{2}$. Na Figura 5, não se observa diferenças significativas nos oscilogramas das soldas feitas com FCAW. Já para o GMAW como visto na Figura 6, percebe-se uma maior irregularidade na maior corrente e que os curtos-circuitos ficaram mais longos, com menor freqüência, como quantificados na Tabela 3. Este é um comportamento atípico, pois se esperava que maiores correntes (proporcionalmente maiores taxa de fusão) resultassem numa freqüência maior e não num maior tempo de curto. Mas este fato é justificável, pois ao se corrigir o comprimento do arco, através do aumento da tensão, para maiores correntes, alcançou-se a maior taxa de fusão com menor frequiência pelo destacamento de gotas maiores na transferência antes de destacar (baseado na redução da frequiência de transferência e pelo cálculo do diâmetro teórico das gotas). Pode-se agora imaginar também que quanto menor a relação de tempo de arco aberto $\left(\mathrm{t}_{\mathrm{ab}}\right)$ sobre tempo de curto-circuito $\left(\mathrm{t}_{\mathrm{cc}}\right)$, menor a emissão de fumos. $\mathrm{Ou}$ seja, assim, os resultados na Tabela 3 corroboram a menor taxa de geração de fumos em GMAW com aumento da corrente para a dada condição. Ou seja, neste caso particular, uma diferença significativa no modo de transferência levou a uma condição onde a massa de fumos não cresce proporcionalmente com o aumento da taxa de fusão para o processo GMAW, como o foi para o FCAW.

Verifica-se ainda na Figura 4 que para o arame tubular com a mistura $\mathrm{Ar}+25 \% \mathrm{CO}_{2}$ se gerou uma TGF em $\mathrm{mg} / \mathrm{kg}$ significativamente menor do que com o gás de proteção $100 \%$ $\mathrm{CO}_{2}$, para os dois níveis de corrente. Este resultado era esperado frente ao maior poder de oxidação da mistura mais rica em $\mathrm{CO}_{2}$. Já o arame maciço também apresentou um aumento na taxa de fumos quando utilizada a proteção com $100 \% \mathrm{CO}_{2}$ no nível de corrente de $150 \mathrm{~A}$. Porém, para o nível de corrente de $250 \mathrm{~A}$, o que se verificou foi uma redução da TGFem $\mathrm{mg} / \mathrm{kg}$ quando utilizado como gás de proteção $100 \% \mathrm{CO}_{2}$ contrariando o esperado pela literatura[2,4]. Acredita-se que a mesma justificativa encontrada para o gás de proteção $100 \% \mathrm{CO}_{2}$ (menor tempo de arco aberto)

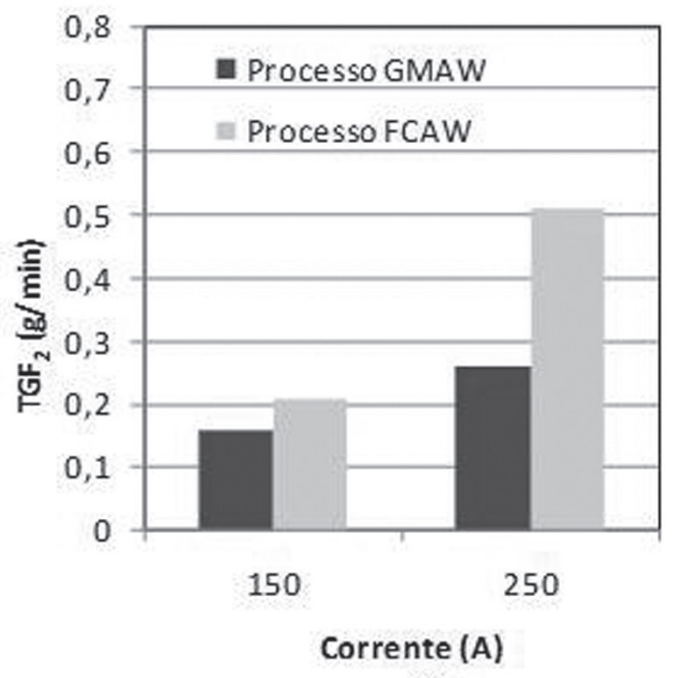

(a) se aplique ao gás de proteção $25 \% \mathrm{CO}_{2}$.

Tabela 3. Valores médios medidos para freqüência de transferências de gotas $\left(\mathrm{F}_{\mathrm{cc}}\right)$, tempos de arco aberto $\left(\mathrm{t}_{\mathrm{ab}}\right)$, tempos de curto-circuito $\left(\mathrm{t}_{\mathrm{cc}}\right)$ e os valores calculados da razão entre esses tempos e o tamanho teórico da gota $\varnothing_{\text {gota }}$ em transferência para as soldagens GMAW

\begin{tabular}{|l|c|c|c|c|c|}
\cline { 2 - 6 } \multicolumn{1}{c|}{} & $\begin{array}{c}\mathrm{F}_{\mathrm{cc}} \\
(\mathrm{Hz})\end{array}$ & $\mathrm{t}_{\mathrm{cc}}(\mathrm{s})$ & $\mathrm{t}_{\mathrm{ab}}(\mathrm{s})$ & $\mathrm{t}_{\mathrm{ab}} / \mathrm{t}_{\mathrm{cc}}$ & $\begin{array}{c}\emptyset_{\text {gota }} \\
(\mathrm{mm})\end{array}$ \\
\hline $100 \% \mathrm{CO}_{2} / 150 \mathrm{~A}$ & 66,23 & 0,0032 & 0,012 & 3,75 & 1,61 \\
\hline $100 \% \mathrm{CO}_{2} / 250 \mathrm{~A}$ & 39,52 & 0,0061 & 0,020 & 3,28 & 3,05 \\
\hline
\end{tabular}

$\mathrm{Na}$ verdade as análises realizadas anteriormente para o GMAW demonstram que o tipo de transferência metálica (embora, para os dois níveis de corrente, o modo de transferência seja por curto-circuito, porém diferenciada) apresentou um maior efeito sobre a TGF em $\mathrm{mg} / \mathrm{kg}$ (taxa relativa de fumos em relação ao material fundido) do que parâmetros como corrente e gás de proteção, mas isso sob a óptica de massa de fumo gerada em função de material fundido. Neste caso é facilmente compreensível a possibilidade da taxa de emissão de fumos não crescer com a mesma razão da taxa de fusão do consumível, o que não aconteceria ao se expressar TGF em $\mathrm{g} / \mathrm{min}$, que representa a taxa absoluta de fumos gerados. Pela Figura 7 verifica-se novamente a maior geração de fumos pelo arame tubular (maior exposição do soldador à taxa de fumos em função do tempo de soldagem), verifica-se ainda que para ambos os processos a taxa de emissão em $\mathrm{g} / \mathrm{min}$ cresce com o aumento da corrente e com a mudança do gás para $100 \% \mathrm{CO}_{2}$.

Mas outro aspecto de suma importância para este estudo exploratório sobre a geração de fumos é a analise sob a óptica de exposição do soldador a estes fumos gerados. Tal análise pode ser feita com ajuda da Figura 8, por meio das taxas que expressam os valores de massa de fumo gerado por unidade de

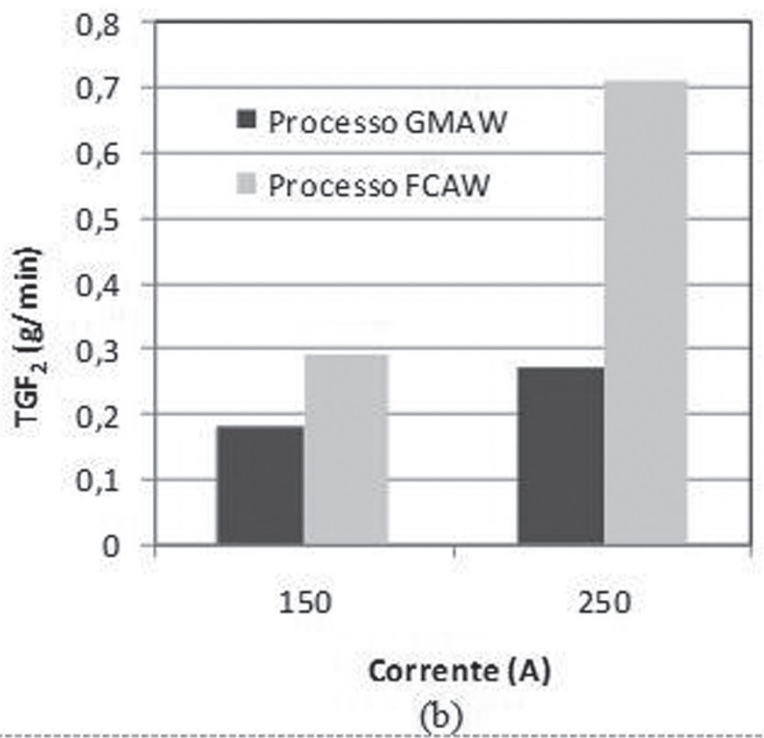

Figura 7. Comparação da $\mathrm{TGF}_{2}(\mathrm{~g} / \mathrm{min})$ para o processo de GMAW e FCAW: (a) para gás de proteção Ar + 25\% CO2; e (b) para gás de proteção $100 \% \mathrm{CO}_{2}$ 


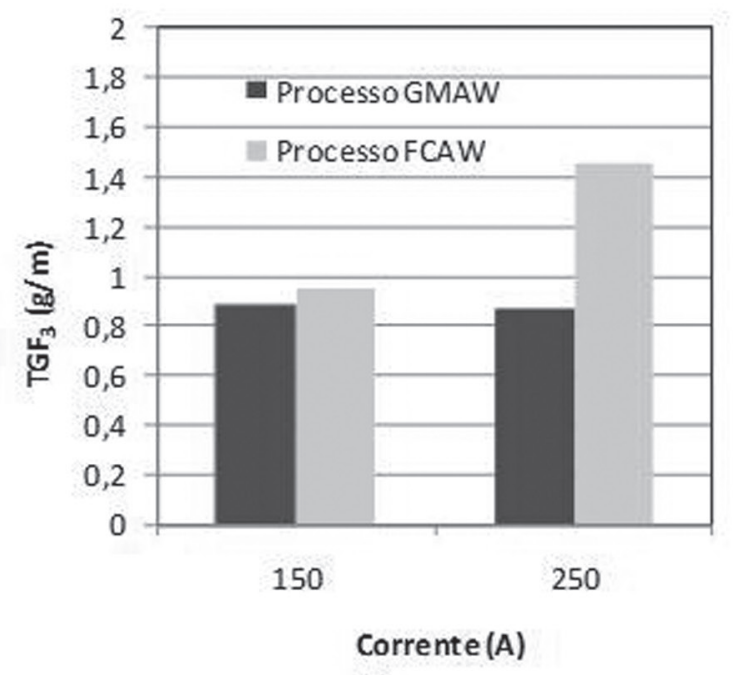

(a)

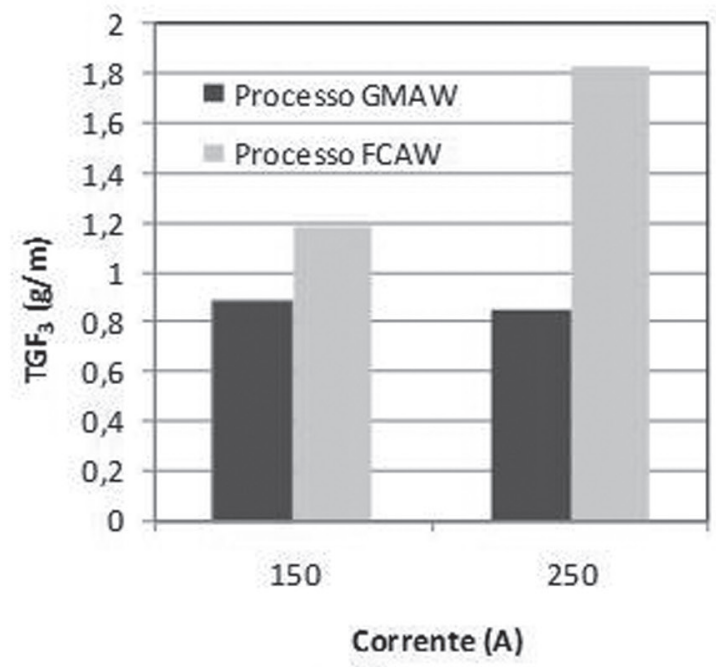

(b)

Figura 8. Comparação da $\mathrm{TGF}_{3}(\mathrm{~g} / \mathrm{m})$ para o processo de GMAW e FCAW: (a) para gás de proteção $\mathrm{Ar}+25 \% \mathrm{CO}$; e (b) para gás de proteção $100 \% \mathrm{CO}_{2}$

Tabela 4. Cálculo da geração da taxa de fumos em função da tensão (valores monitorados e regulados)

\begin{tabular}{|c|c|c|c|c|c|}
\hline Arame & Teste & $\mathrm{U}_{\text {reg }}(\mathrm{V})$ & $\mathrm{I}(\mathrm{A})$ & $\mathrm{U}(\mathrm{V})$ & TGF $(\mathrm{g} / \mathrm{min})$ \\
\hline Tubular & $100 \% \mathrm{CO}_{2}($ Teste 10$)$ & 23 & 142,7 & 23 & 0,29 \\
\hline Tubular & $100 \% \mathrm{CO}_{2}($ Teste 5$)$ & 26 & 152,7 & 25,7 & 0,29 \\
\hline Tubular & $100 \% \mathrm{CO}_{2}($ Teste 9$)$ & 29 & 155,7 & 29,2 & 0,34 \\
\hline Maciço & $100 \% \mathrm{CO}_{2}$ (Teste 12) & 19 & 152,9 & 19,2 & 0,16 \\
\hline Maciço & $100 \% \mathrm{CO}_{2}$ (Teste 2) & 21 & 151,5 & 20,8 & 0,18 \\
\hline Maciço & $100 \% \mathrm{CO}_{2}($ Teste 11$)$ & 23 & 148,2 & 22,9 & 0,18 \\
\hline
\end{tabular}

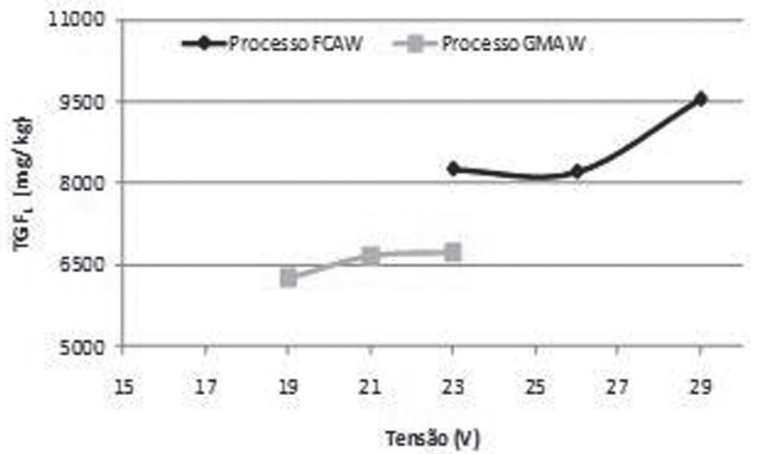

(a)

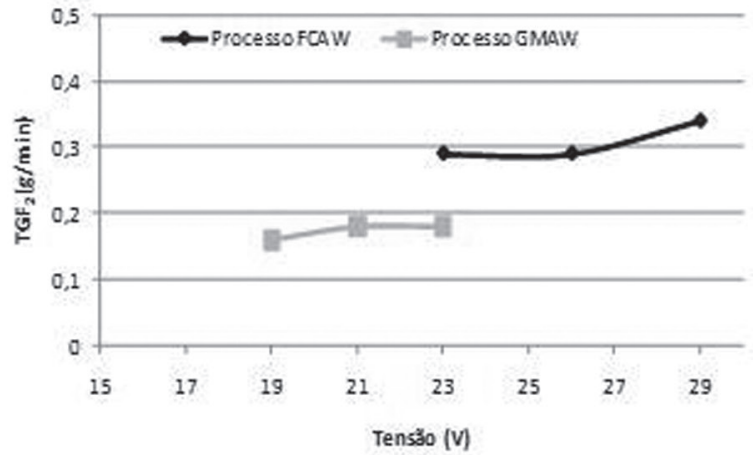

(b)

Figura 9. Taxa de fumos relativa $\mathrm{TGF}_{1}(\mathrm{mg} / \mathrm{Kg})$ e absoluta $\mathrm{TGF}_{2}(\mathrm{~g} / \mathrm{min})$ em função da variação da tensão para o processo FCAW e GMAW

comprimento de solda realizada $\left(\mathrm{TGF}_{3}\right)$. A análise desta figura torna-se interessante porque através da Tabela 1 verifica-se que o arame tubular apresenta velocidades de soldagens superiores às velocidades empregadas para o arame maciço, ou seja, para produzir um mesmo volume de cordão (premissa deste trabalho) o tubular requer menos tempo de soldagem. Porém, verifica-se na Figura 6 que mesmo o arame tubular efetuando soldagens em um tempo menor que o arame maciço, o volume de fumos gerados ainda é maior. Ainda analisando este gráfico, observa-se que novamente o efeito do aumento da corrente e da mudança de gás volta a ser diferente entre FCAW e GMAW. É importante ressaltar que novamente o índice de medida de emissão passa a ser relativo, já que as massas depositadas eram as mesmas para cada arame. E que as mesmas justificativas baseado no efeito da transferência se aplicam aqui.

A Tabela 4 refere-se aos testes realizados para avaliar o efeito da tensão sobre a taxa de geração de fumos. Os testes foram realizados apenas para um nível mais baixo de corrente e um 
tipo de gás de proteção $\left(100 \% \mathrm{CO}_{2}\right)$, usando-se para regulagem da tensão, além do valor médio da faixa de estabilidade dos dois processos para as dadas correntes, os valores limites desta faixa (desta forma, mesmo que com variação de tensão, a mesma foi aplicada em condições que se poderia usar na prática).

A Figura 9 enfatiza que o processo FCAW gera níveis mais altos de taxa de fumos do que o processo GMAW, tanto de forma relativa (ao volume de arame fundido ou depositado) ou absoluta (em termos de tempo). Para o arame tubular, verificase que quando se variou a tensão houve um pequeno aumento desta taxa, mas não muito significativo, mas a variação da tensão de regulagem aconteceu em uma faixa mais larga (6 V contra 4 V). Já para o processo com arame maciço não há variação na taxa de fumos com o aumento da tensão. Estes resultados estão em desacordo com o apresentado na literatura pela Figura 1, mas uma justificativa para tal seria que neste estudo, apesar da variação da tensão, não houve variação no tipo de transferência metálica. Estes resultados, juntamente com os anteriores, sugerem que o tipo de transferência tem forte influência sobre a geração de fumos, mais até do que a corrente.

\section{Conclusões}

A partir das condições (mesmo volume de cordão depositado a uma mesma corrente, com regulagem de distância bico de contato peça, coerente com o especificado para o produto) e materiais (AWS ER70S-6 e E71T-1) estudados, confirmou-se o esperado que há uma maior taxa de geração de fumos pelo processo FCAW (arame tubular) do que com o GMAW (arame maciço), o que confirma a adequação da metodologia usada.

Mas resultados não tão previsíveis foram obtidos pela quantificação da taxa de fumos sob duas perspectivas, a do consumível em si como fonte de geração de fumos e sob a óptica de exposição do soldador aos fumos gerados. Foi verificado que: a) Tanto um aumento da corrente (na verdade, da taxa de fusão do arame) como o uso do gás de proteção mais ricos em $\mathrm{CO}_{2}$ favorecem a geração de fumos, a menos que outro efeito concorrente os superem;

(b) A taxa de geração de fumos é fortemente influenciada pelas características da transferência metálica (volume da poça, frequiência de transferência, tempo de arco aberto, etc.), podendo até superar o efeito da corrente e/ou gás de proteção (na verdade, o efeito da taxa de fusão do arame) e do gás de proteção, como aconteceu com o processo GMAW;

(c) A variação da tensão dentro da faixa parametrizada de uso adequado do processo (sem alterar o modo de transferência), tanto para o processo com arame maciço quanto para o processo com arame tubular, não demonstrou alterar a taxa de emissão de fumos;

(d) A maior capacidade de produção do arame para FCAW usado neste trabalho, que permitiu a confecção de o mesmo volume depositado de cordão em menor tempo, fez diminuir a exposição do soldador aos fumos em comparação ao arame maciço usado no GMAW, mas ainda assim o arame tubular leva a uma maior exposição de fumos.

É importante destacar que estes resultados só podem ser considerados para os produtos comercias avaliados. Ou seja, só se poderia afirmar de forma genérica que o processo com arame tubular geraria maior quantidade de fumos do que o processo com arame maciço, para depositar uma mesma quantidade de material, se mais arames fossem avaliados. Além disto, uma geração maior de fumos não implica necessariamente em um maior poder de contaminação do ambiente, inclusive no que se refere ao cumprimento de normas de saúde ocupacional, pois se precisaria analisar o tipo de fumos.

\section{Agradecimentos}

Os autores agradecem a todos que contribuíram para o desenvolvimento do trabalho. Em especial ao CNPq pelo fornecimento de bolsa de estudo/pesquisa aos autores e à Lincoln pelo fornecimento do arame tubular.

\section{Referências Bibliográficas}

[1] ANTONINI J. M.; MURTHY G. G. K. How welding fumes affect the welder. Welding journal 1998, vol. 77, nº10, p. 5559 .

[2] CASTNER, H.R. Gas Metal Arc Welding Fume Generation using Pulsed Current, Welding Journal, 1995, vol. 74, n², p. 59-68.

[3] QUIMBY, B.J.; ULRICH G.D. Fume Formation Rates in Gas Metal Arc Welding. Welding Journal, Apr 1999, p. 142-149. [4] YAMAME, K. Low Fume Types of Welding Materials improve the Welding Environment. Welding International, vol $21, \mathrm{n}^{\circ} 5,2007$, p. $337-346$.

[5]LANCASTER, J. F. The Physics of Welding. $2^{\circ}$ ed, Oxford, Pergamon Press, $1986340 \mathrm{p}$.

[6]JENKINS, N.T.; MENDEZ, P.F.; EAGAR, T.W.; Effect of Arc Welding Electrode Temperature on Vapor and Fume Composition. Proceedings of the Gas Metal Arc Welding for the 21 st Century Conference; Orlando, FL, Dec 6-8, 2000, p. 2. [7]GRAY, C.N., HEWITT, P. J., and DARE, P.R. M, 1982. New approach would help Control Welding Fumes at source part two: Mig Fumes .Welding and Metal Fabrication, October, pp. 393-397 apud QUIMBY, B.J.; ULRICH G.D. Fume Formation Rates in Gas Metal Arc Welding. Welding Journal, Apr 1999 p. 142-149.

[8] STARLING, C.M.D; MODENESI, P.J. Caracterização do Cordão na Soldagem FCAW com um Arame Tubular Básico. Soldagem e Inspeção, vol 13, no 4, 2008, p. 304-318.

[9] ROSADO, T., PIRES I., QUINTINO L. Opportunities to Reduce Fume Emissions in Gas Metal Arc Welding, ISQ-doc. VIII-2094-09.

[10] AWS F1.2:2006 An American National Standard; Laboratory Method For Measuring Fume Generation Rates and Total Fume Emission of Welding and Allied Processes.

[11] ASTM C800, American Society for Testing and Materials, Standard Specification for Fibrous Glass Blanket Insulation (Aircraft Type), outubro de 2008, p. 1-5.

[12]FERNANDES, D.B.; SCOTTI A. Procedimento para o Ensaio Laprosolda de Geração de Fumos em Soldagem MIG/ MAG 14/2009, versão 9-1, p. 1-12. 